Proc. of the XI Int. Conf. - Ion Implantation and other Applications of Ions and Electrons, Kazimierz Dolny 2016

\title{
Effect of Sample Thickness on Carbon Ejection from Ultrathin Graphite Bombarded by $\mathrm{keV} \mathrm{C}_{60}$
}

\author{
M. Golunski and Z. Postawa* \\ Institute of Physics, Jagiellonian University, S. Łojasiewicza 11, 30-348 Kraków, Poland
}

\begin{abstract}
Molecular dynamics computer simulations are employed to investigate the effect of a sample thickness on the ejection process from ultrathin graphite. The thickness of graphite varies from 2 to 16 graphene layers and the system is bombarded by $10 \mathrm{keV} \mathrm{C}_{60}$ projectiles at normal incidence. The ejection yield and the kinetic energy of emitted atoms are monitored. The implications of the results to a novel analytical approach in secondary ion mass spectrometry based on the ultrathin free-standing graphene substrates and transmission geometry are discussed.
\end{abstract}

DOI: 10.12693/APhysPolA.132.222

PACS/topics: Computer simulations, sputtering, graphene

\section{Introduction}

In recent years cluster ion beams have attracted increasing experimental and theoretical attention due to their capacity to enhance the ejection of large intact organic molecules in secondary ion mass spectrometry (SIMS) $[1,2]$. One of the most successful clusters used in organic SIMS is $\mathrm{C}_{60}$ fullerene [3]. In the typical SIMS geometry the detector is located at the same side of the target as the ion gun. Usually metal or semiconductor supports are used to deposit the investigated material. A novel SIMS configuration, using transmission orientation, has been proposed recently by a group from Texas A\&M University [4]. In this orientation, the analysed organic material is deposited at one side of the ultrathin substrate, while another side is bombarded by cluster projectiles. It is argued that such geometry can be particularly attractive for the analysis of small amounts of organic material, molecular nano-objects and supramolecular assemblies.

So far only one simulation has been done for $\mathrm{C}_{60}$ bombardment of graphene system [4]. Most of the existing simulations are performed on thick graphite [5-11]. Moreover, many of these studies concentrate on defect creation in the bombarded system rather than on material ejection. Theoretical studies of graphite sputtering by $\mathrm{keV} \mathrm{C}_{60}$ projectiles show that the sputtering yield is unexpectedly low $[5,6]$. Krantzman et al. attribute this fact to a low atomic density of graphite [5] while the effect of layered structure of graphite was emphasised by Tian et al. [6]. It has been also shown that the membrane-like structure of graphite can be made to vibrate as a result of a low-energy cluster impact $[4,9]$. The mesoscopic motion of created low-energy circular acoustic waves can stimulate ejection of small weakly bound organic molecules adsorbed at graphite. It has been found that large molecules can be ejected from metal or semiconductor substrates by simultaneous and correlated collisions with

*corresponding author; e-mail: zbigniew.postawa@uj.edu.pl ejecting substrate atoms $[12,13]$ or by energetic deformations occurring during crater unfolding $[1,14,15]$. We would like to test if a similar phenomenon can be observed at the ultrathin graphite.

The goal of this paper is, therefore, to supply theoretical description of processes that occur in the ultrathin graphite systems of various thickness bombarded by the $\mathrm{keV} \mathrm{C}_{60}$ projectile. We will concentrate on monitoring ejection of projectile and substrate atoms, testing viability of ultrathin graphite as a substrate for organic analysis.

\section{Computer model}

A detailed description of the molecular dynamics computer simulations used to model cluster bombardment can be found elsewhere [1]. Briefly, the motion of particles is determined by integrating the Hamilton equations of motion. The forces among carbon atoms in our system are described by a Reax-FF force field [16] splined at short distances with a Ziegler-Biersack-Littmark (ZBL) potential to properly describe high energy collisions. The shape and size of the samples are chosen based on visual observations of energy transfer pathways stimulated by impacts of $\mathrm{C}_{60}$ projectiles. As a result, cylindrical samples with a radius of $200 \AA$ are used. Samples with a thickness between 2 and 16 graphene layers with a high oriented pyrolytic graphite (HOPG) structure are bombarded by $10 \mathrm{keV} \mathrm{C}_{60}$ projectiles that are directed at the bottom of the sample. Rigid and stochastic regions are used to simulate the thermal bath that keeps the sample at required temperature, to prevent reflection of pressure waves from the boundaries of the system, and to maintain the shape of the sample $[1,17]$. The simulations are run at $0 \mathrm{~K}$ target and extend up to $4 \mathrm{ps}$, which is long enough to achieve saturation in the sputtering yield vs. time dependence. Sixty four randomly selected impact points located near the center of the sample are chosen to achieve statistically reliable data.

\section{Results and discussion}

Cross sectional views of the temporal evolution of 2,8 , and 16-layer systems bombarded by $10 \mathrm{keV} \mathrm{C}_{60}$ projectiles are shown in Fig. 1. It is evident that $\mathrm{C}_{60}$ clusters 
fragment into smaller pieces, almost immediately after the impact. At the 2-layer (2L) sample almost all projectile atoms penetrate through the substrate, as shown in Fig. 2a. Nevertheless, already a half of the primary kinetic energy is transferred to the sample, as shown in Fig. 2b. Most of this energy is carried away by the substrate particles emitted in the transmission direction. No sample atoms are sputtered. The projectile impact leads to a creation of cylindrical acoustic waves that propagate outward from the point of impact with a maximum amplitude of $1 \AA$.

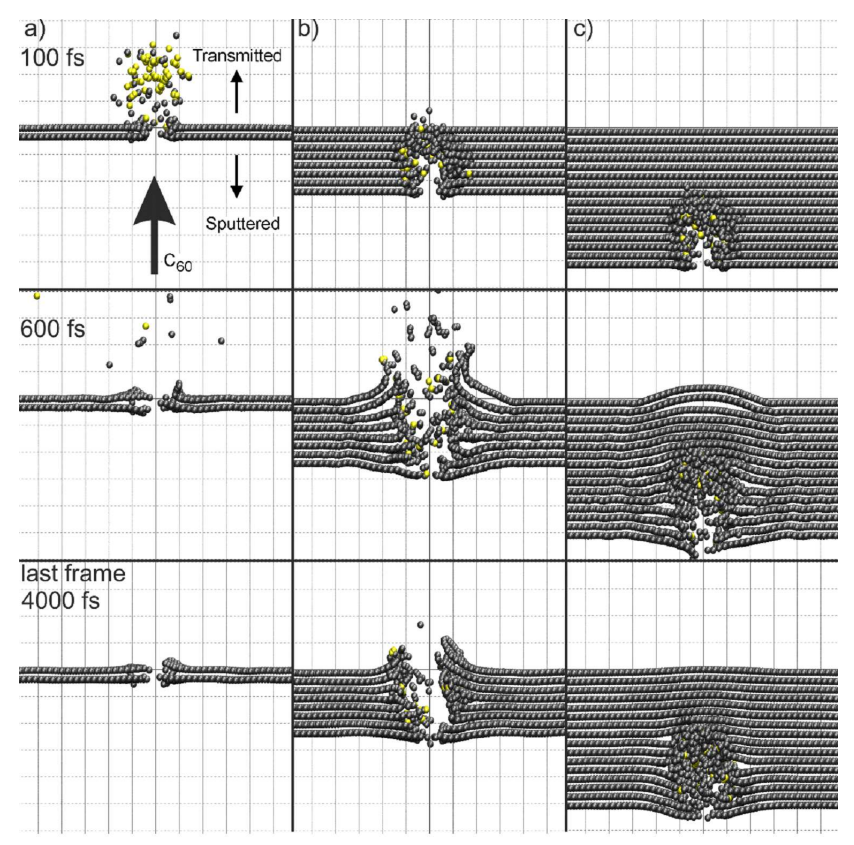

Fig. 1. Cross-sectional view of the temporal evolution of a typical collision event leading to ejection of atoms due to $10 \mathrm{keV} \mathrm{C}_{60}$ bombardment of a system composed of (a) 2, (b) 8, and (c) 16 graphene layers. Bright (yellow) spheres indicate the projectile atoms. A $1.5 \mathrm{~nm}$ wide slice of the system centred at the impact point is shown. The dashed lines in the background are separated by $10 \AA$. The arrows indicate directions of the primary beam, transmitted and sputtered atoms.

Much more dramatic alteration is observed for the $8 \mathrm{~L}$ system. Projectile is more efficiently decelerated, depositing almost all its kinetic energy into the sample. As a result, the energized cylindrical volume is created along the projectile path. Bonds of many carbon atoms located in this volume are broken which means that these atoms are highly reactive. Soon after the projectile impact sample integrity is compromised. Graphene sheets are bent up in the direction of moving projectile and, for a few hundred fs, they are even separated from each other near the point of impact. Finally, cylindrical opening is formed in the sample. It is surrounded by an elevated rim at the top surface of the sample. No rim is formed at the bottom surface. The wall of the opening is hardened by interlayer new bonds that form between under-coordinated carbon atoms.
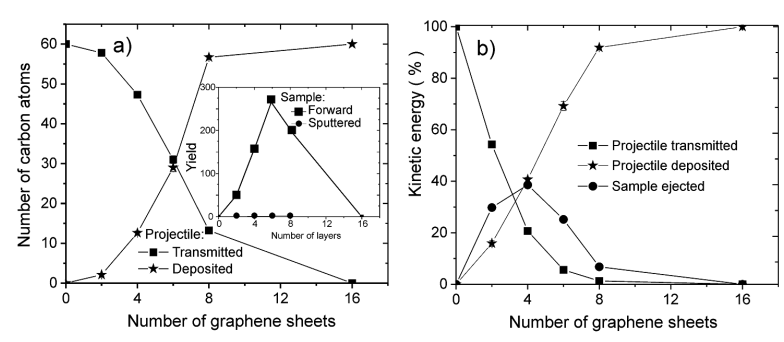

Fig. 2. Dependence of (a) the number of transmitted and sample-deposited projectile atoms and (b) the percentage of the primary kinetic energy carried by the transmitted projectile atoms, ejected sample atoms, as well as the energy deposited in the sample (projectile deposited) on the number of graphene sheets for $10 \mathrm{keV}$ $\mathrm{C}_{60}$ projectiles at normal incidence. Inset to part (a) shows dependence of a number of sample atoms ejected in the forward and sputtered directions on the sample thickness.

All bombarded systems, except for 16-layer graphite, are perforated by a $\mathrm{C}_{60}$ projectile. The number of projectile atoms penetrating the substrate decreases with a thickness of the sample, as indicated in Fig. 2a. At the same time, the number of projectile atoms getting implanted in the sample increases in the inverse way, as fewer than one projectile atom is backreflected on average. The sputtering yield is almost zero for all investigated systems, as shown in the inset to Fig. 2a. It has been proved that the layered structure of graphite is mostly responsible for a lack of sputtering [6]. Computer simulations indicate that the energy transfer in graphite is highly anisotropic and occurs predominantly along graphene sheets. Not much energy is transferred in the vertical direction. Consequently, energy that normally would be directed towards the surface is now laterally channelled and does not contribute to particle ejection.

Despite a very low sputtering yield, the ejection of substrate atoms in the transmission direction can be quite significant. These particles are ejected by collisions with the projectile atoms very soon after the projectile impact before the deposited energy is effectively drained out of the altered volume. As shown in the inset of Fig. 2a, the dependence of the ejection yield on the substrate thickness is non-monotonic. At first, the signal increases as more carbon atoms become available for ejection when the sample is getting thicker. However, at the same time more primary kinetic energy has to be sacrificed to penetrate through a thicker solid. As a result, less energy is available near the surface from where the ejection occurs and ultimately the overall signal decreases.

Our results indicate that such ultrathin graphite substrates supports can have several advantages over the traditional metal or semiconductor substrates for analysis of small amounts of organic material. Firstly, the extremely small thickness of the support results in small amounts of emitted substrate material. As a result, there is a minimal interference between the substrate and the analyzed signal. From this point of view the $2 \mathrm{~L}$ system is the most optimal. In this system also a large portion of the 
primary kinetic energy can be transmitted to the organic overlayer in the direction towards the detector, increasing a chance that a small amount of analyte can be recorded. However, the energetics of this transfer is also important. In a $2 \mathrm{~L}$ system both projectile and sample atoms ejected in the transmission direction have high kinetic energies. A rough estimate based on the data presented in Fig. 2 implies that the average kinetic energy of these atoms is around $100 \mathrm{eV}(\approx 5500 \mathrm{eV} / 58$ atoms $)$ for the projectile and $60 \mathrm{eV}(\approx 3000 \mathrm{eV} / 50$ atoms $)$ for the sample atoms. These values are several times larger than a bond energy in organic molecules. As a result, the molecules that will be hit by these atoms will be fragmented. Apart from ejection of atoms, there is not much movement present in the $2 \mathrm{~L}$ system. The energy of acoustic waves is admittedly sufficient to eject small molecules but will not be sufficient to eject a massive particle. All these arguments indicate that $2 \mathrm{~L}$ systems may not be the best choice.

Much more promising is the $8 \mathrm{~L}$ system. As shown in Fig. 1b, unfolding of the topmost graphene sheet can work as a catapult that will hurl large molecules adsorbed in this region into the vacuum. Such phenomenon is one of the processes responsible for ejection of large molecules from the metal substrates where unfolding of the crater rims serves as a catapult $[1,14,15]$. There is a considerable amount of energy associated with this movement which means that even very large molecules can be uplifted. However, in graphite this movement extends to a much larger lateral distance from the point of impact as compared to the analogous phenomenon present in metals $[1,14,17]$. As a result, this mechanism will be more effective in ultrathin graphite. A larger number of adsorbed molecules can be ejected by a single projectile impact making analysis of small amounts of organic material viable. Catapult-like ejection of organic molecules can be additionally enhanced by correlated collisions with ejecting substrate atoms $[12,13]$. As can be deduced for Fig. 2 many substrate atoms are ejected with the average kinetic energy of $3 \mathrm{eV}$ which is low enough not to fragment molecules. As shown in Fig. 1c, perforation of ultrathin graphite is not necessary to stimulate molecular emission. The upper surface of the $16 \mathrm{~L}$ substrate buckles up during the projectile deceleration. This motion potentially can also cause ejection of large molecules that are physisorbed at the upper surface.

\section{Conclusions}

Molecular dynamics computer simulations have been performed to study the effect of the sample thickness on the ejection efficiency of particles emitted from the ultrathin graphene layers bombarded by $10 \mathrm{keV} \mathrm{C}_{60}$ projectiles. The number of transmitted projectile atoms decreases with the thickness of the sample. All atoms that are not transmitted are being implanted in the sample as almost no backreflection is present due to a lack of vertical motion in the system and strong covalent $\mathrm{C}-\mathrm{C}$ bonds. The dependence of a number of sample atoms ejected in the transmission direction on the layer thickness has a maximum at a four layer system. Such behavior can be explained by an interplay between the amount of material available for ejection and the energy available near the surface from where ejection occurs. No sputtering is observed which is attributed to a lower atomic density and the layered structure of graphite. It has been shown that ultrathin graphite can be an interesting support for organic SIMS where a small amount of organic material is probed.

\section{Acknowledgments}

The authors gratefully acknowledge financial support from the Polish National Science Center, Program No. 2013/09/B/ST4/00094 and 2015/19/B/ST4/01892.

\section{References}

[1] B.J. Garrison, Z. Postawa, Mass Spectrom. Rev. 27, 289 (2008).

[2] N. Winograd, Anal. Chem. 77, 142A (2005).

[3] D. Weibel, S. Wong, N. Lockyer, P. Blenkinsopp, R. Hill, J.C. Vickerman, Anal. Chem. 75, 1754 (2003).

[4] S.V. Verkhoturov, S. Geng, B. Czerwinski, A.E. Young, A. Delcorte, E.A. Schweikert, J. Chem. Phys. 143, 164302 (2015).

[5] K.D. Krantzman, R.P. Webb, B.J. Garrison, Appl. Surf. Sci. 255, 837 (2008).

[6] J.T. Tian, T. Zheng, J.Y. Yang, S.Y. Kong, J.M. Xue, Y.G. Wang, K. Nordlund, Appl. Surf. Sci. 337, 6 (2015).

[7] M. Kerford, R.P. Webb, Nucl. Instrum. Methods Phys. Res. B 153, 270 (1999).

[8] M. Kerford, R.P. Webb, Nucl. Instrum. Methods Phys. Res. B 180, 44 (2001).

[9] R.P. Webb, Radiat. Eff. Def. Solids 162, 567 (2007).

[10] R.P. Webb, M. Kerford, M. Kappes, G. Brauchle, Radiat. Eff. Def. Solids 142, 23 (1997).

[11] C. Anders, H. Kirihata, Y. Yamaguchi, H.M. Urbassek, Nucl. Instrum. Methods Phys. Res. B 255, 247 (2007).

[12] B.J. Garrison, A. Delcorte, K.D. Krantzman, Acc. Chem. Res. 33, 69 (2000).

[13] R. Chatterjee, Z. Postawa, N. Winograd, B.J. Garrison, J. Phys. Chem. B 103, 151 (1999).

[14] Z. Postawa, Appl. Surf. Sci. 231, 22 (2004).

[15] B. Czerwinski, R. Samson, B.J. Garrison, N. Winograd, Z. Postawa, Vacuum 81, 167 (2006).

[16] L.C. Liu, Y. Liu, S.V. Zybin, H. Sun, W.A. Goddard, J. Phys. Chem. A 115, 11016 (2011).

[17] Z. Postawa, B. Czerwinski, M. Szewczyk, E.J. Smiley, N. Winograd, B.J. Garrison, Anal. Chem. 75, 4402 (2003). 